\title{
Creation of Geodistributed SCADA-System
}

\author{
P.A. Kabanov ${ }^{1}$, M.S. Sukhodoev ${ }^{1}$ \\ Peter96pvl@gmail.com|smike@ tpu.ru \\ ${ }^{1}$ Tomsk Polytechnic University, Tomsk, Russia
}

This article shows the possibility of combining modern software development technologies. SCADA systems usually do not use the full power of modern visualization technologies (rendering of three-dimensional objects, spatial transformation, etc.). In addition to graphics, users of these systems have a question about the possibility of using GIS systems to facilitate the determination of the location of objects (on global, local, and other maps). Using all the technologies described in this article, a system was developed that meets the basic requirements of the SCADA system. The developed system perfectly combines three-dimensional visualization and support of the GIS system. These implementations showed excellent results and feedback from users (with the test and final versions of the program).

Keywords: development, programming, SCADA systems, GIS, computer graphics, C\#, ESRI, 3D, ArcGIS, SQL.

\section{Introduction}

Currently, in addition to the typical tasks carried out by the SCADA-systems, such as maintaining history, continuous monitoring and control of remotely connected equipment, there was a need to create geographic schemes with the image of engineering objects on the map. In some cases, it is required that the engineering objects on the map are not just pictures, but selectable 3D objects that have their properties, functions of interaction with the user and support for opening mimic diagrams of substations traditional for SCADA systems.

The scientific novelty of the development is that the combination of GIS, SCADA and 3D visualization technologies positively affects the perception of user information, improves overall performance, and also allows you to solve a wider range of tasks that a specialist faces.

The combination of three technologies allows you to solve various engineering problems that are associated with these technologies. Thus, you can accurately know the location of the equipment, its configuration and instrument reading. There is an opportunity to consider problem areas on a large and small scale. It also allows you to use the program not only for a certain circle of specialists, but also for demonstration and reporting to people who have less competence.

Actual now, the development of software complexes for remote monitoring and dispatching equipment control at geographically remote sites is needed. Which represents an integrated solution that combine the capabilities of several systems - Geoinformation System (GIS), SCADA and ACS. The most promising, in this case, is the implementation of the SCADA-system interface based on web technologies.

During the development and testing of the program, it was revealed:

A better perception of information and understanding of the problem area when combining instrument readings with their 3D models.

Performance improvement in reporting, which includes the geographical data of the location of the equipment, as well as a description of this equipment.

The main idea of this article is to develop a system that will meet the requirements, which will be discussed later in this article.

\section{Main tasks}

The development task was the creation of a Windows desktop application with 3D graphics and online viewing of maps support, as well as images on the map, their location, information and support for a quick jump to the overview of objects.

SCADA is a software package designed to develop or provide a real-time operation of systems for collecting, processing, displaying and archiving information about object monitoring or control $[1,10]$. This software is installed on computers and uses I/O drivers or OPC / DDE servers for communication with the object. The program code can be either written using one of the programming languages, or is generated by the design environment.

The main tasks $[4,10]$ solved by SCADA-systems are:

1. Data exchange between the "communication devices and the object" (with industrial controllers and I/O cards) in real time via drivers.

2. Real time data processing.

3. Logical control.

4. Display information on the monitor screen in a convenient and human-readable form.

5. Maintenance of a real-time database with technological information.

6. Alarm and alarm management.

7. Preparation and generation of reports on the progress of the technological process.

8. The introduction of network communication between the SCADA and PC.

9. Providing communication with external applications (DBMS, spreadsheets, word processors, etc.).

GIS - is a system for collecting, storing, analyzing and graphically visualizing spatial data and associated information about the required objects. Geoinformation system can include in its composition spatial databases (including under the control of universal DBMS), editors of raster and vector graphics, and various means of spatial data analysis, which are applied in cartography, geology, meteorology, land management, ecology, municipal administration, transport, economy, defense and many other areas. Scientific, technical, technological and applied aspects of designing, creating and using geoinformation systems are studied by geoinformatics.

\section{Development}

One of the most powerful GIS-systems - "ArcGIS" was chosen for the SCADA system development. ArcGIS is the family of geoinformation software products of the American company ESRI. They are applied for land cadasters, land management, accounting of real estate objects, engineering communications systems, geodesy, subsoil use and other areas.

Next, we will consider the architecture of the developed SCADA system. The application itself consists of three main windows in each of which there are basic modules and submodules. The application is connected directly to the SQL database, from which it takes all the necessary information about the objects. It also interacts with ArcGIS-online to correctly display geo -information on the map.

The application was created as an alternative SCADA system for gas transmission system facilities. As an example, one of several objects was taken. 
The main development environment was Microsoft Visual Studio Enterprise. The main programming language (C\#) and the markup language $(\langle\mathrm{XAML}\rangle)$ were used, since the application was made on the WPF platform (Windows Presentation Foundation) $[2,7,9]$.

This application is an example of the possibilities of modern development of SCADA-systems in the current programming languages using all the newest technologies. All the features that are implemented in the application can be transferred, improved and used in various industries, or on other similar objects.

The application consists of three windows, which we consider below.

The first window consists of two main modules, one submodule and control buttons (Fig. 1).

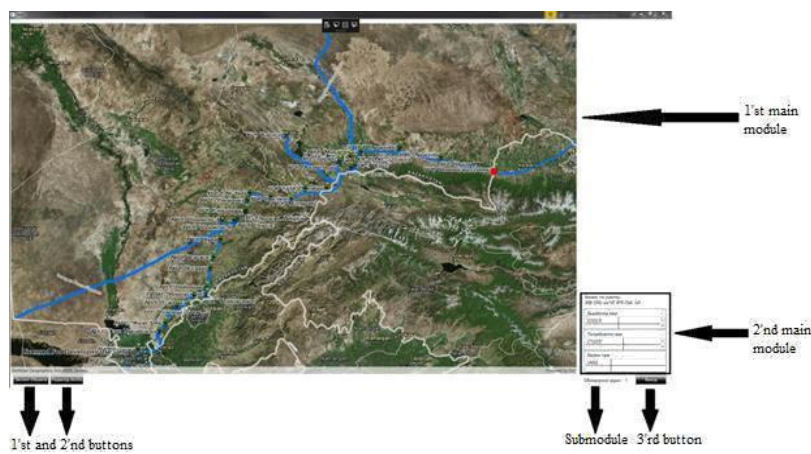

Fig. 1. Scheme of application's main window.

First button (name: "detail view") - is the button for detailed consideration of the object (this will be described below in a note). That button can move user to the next level of the program (2nd window).

Second button (name: "run ArcGIS") - is the button that enables the ArcGIS editor [3]. It allows you to open the GIS system editor and make the necessary adjustments if ArcGIS Desktop is installed on PC (the package consists of ArcGlobe, ArcCatalog, ArcMap, ArcScene) and the application package has been updated.

Third button (Name: quick exit) closes the user's environment.

First main module is an online map [5, 6, 8]. It is a display module with information about objects from the database (see Fig. 1). The mode and color scheme of the map is configured directly in ArcGIS-online, so that the user with the account can easily customize everything. The map itself contains a part of Kazakhstan's gas transmission system; objects located on this system; information about objects; names and signatures of objects; built-in event handler for the user's work with the mouse (full interactivity: scrolling by scrolling, movement on the map and response to pressing the left mouse button); button that is located on the selected object and serves for a quick transition to the next level of the program (this will be described below in a note). All objects and elements were located by exact geodata and coordinates.

Second module (see Fig. 1) is the module for calculating balances on the selected section of the gas transportation system. Balances are displayed on the sections indicated on the map.

Single submodule (see Fig. 1) updates information that are coming from the database every 10 seconds (this time was chosen for complete synchronization of incoming information). For convenience, the time until the update is displayed on the display, and the user knows when the actual information will arrive.

Note - this feature was displayed both as a button on the map, and as a control button, for adaptation and convenience to the user of the program.
The second window consists of one main module, a submodule and two control buttons (see Fig. 2).

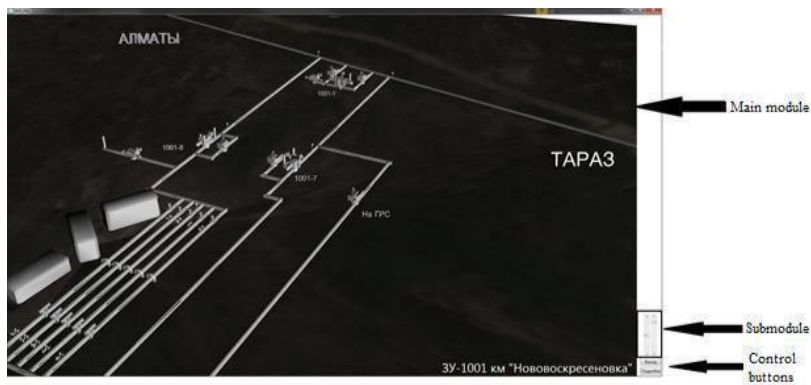

Fig. 2. Scheme of application's second window.

First button (name: "to map view") moves back to the previous window.

Second button (name: "to detailed view") moves to the next window.

First main module (see Fig. 2) was created using the technology Viewport3D, built-in WPF. This technology allows you to work, demonstrate and edit 3D objects in a desktop application. With this technology, the selected object can be recreated, with the ability to view it. The arrangement of all elements is the same as in the real world. This realism was achieved by digitizing images from the satellite and photos in 3Ds Max with further transfer to Viewport3D. This module is fully interactive and responds to mouse clicks (moving behind the cursor), and scrolls the mouse wheel (zooms in and out of the camera).

Single submodule (see Fig. 2) contains two scrollbars, which are responsible for controlling the camera in the main module. The first reacts the same way as on the mouse wheel (bring closer and detaches the camera). The second submodule allows to rotate the entire object around its axis and consider all the elements at different angles.

The third window consists of one main module, four submodules and three control buttons (see Fig. 3).

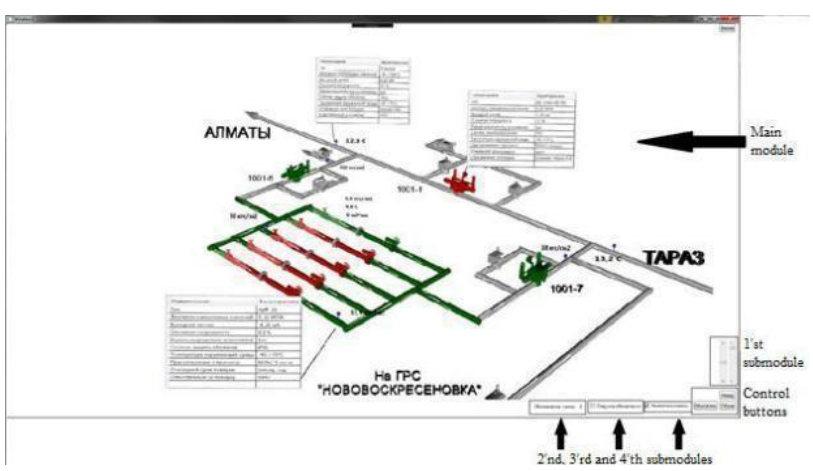

Fig. 3. Scheme of application's third window.

First button (name: "to previous view") returns the user to the previous window.

Second button (name: "to map view") returns the user to the main screen.

Third button (name: "quick exit") closes the program.

One main module - is a 3D scheme of the object with all the elements displays the state of the cranes in real time (on / off), and this object accordingly changes color when updating the database (green / red). In addition, the branches of the gas pipelines depend on the state of the cranes and change the indicators (active / inactive) and the color (green / red), when the state of the cranes changes and the database is updated. Some elements of the scheme are interactive. When you click them, the windows with the passport data for the item are displayed, the second time they disappear. 
Next to certain sensors, digital data on temperature, pressure and other information are displayed. If sensors or gas lines are not active, no data is displayed. There are corresponding signatures of sensors, elements, threads of directions, as well as units of measurement. This module is completely dependent on the submodules of this window, and shows all the most basic functions when working with the application.

First submodule (see Fig. 3) consists of a checkbox that allows you to enable or disable freezing (the ability to work) cameras (it is enabled by default). When the freeze is turned off it is possible to work with the camera as on the second window (the camera scales, reaction to pressing the left mouse button). It is also interconnected with the 2 submodules. Second submodule (see Fig. 3) consists of two scrollbars, which allow working with the camera of the main module. Thefirst rotates the object around its axis and makes it possible to view

About the authors it at various angles. The second shows the five main camera positions ( 1 is the top-to-right position, 2 is the central-top position, 3 is the left-top position, 4 is the top-down or 2D-view, position "detailed consideration of the element"). Interconnected with first submodule: if the camera is unfrozen, the first scroller of camera rotation works; if the camera is frozen, then the second scrollbar of the camera's main positions is operating.

Third submodule (see Fig. 3) is the submodule for updating values from the database. It operates the same as the first window. It displays the count until all values are updated.

Fourth submodule consists of a checkbox that allows you to enable or disable the voice notification of a change in the state of the element. When enabled, it notifies the user about updating the database, or about changing the state of certain items. It works with a script for Speech Application Programming Interface or Microsoft Speech API.

The C\# was used to write the logic of interaction between windows, their elements and event handling.

The $<$ XAML $>$ was used to design the external user interface and 3D-graphics.

This application is supported on Windows (7, 8.1, and 10) with the .Net Framework 4.6.1 and higher.

\section{Conclusion}

The developed demo version of the application meets the following main requirements $[4,10]$ of the SCADA system:

1. Data exchange with "communication devices with the object" (with industrial controllers and I/O cards) in real time via drivers.

2. Real time data processing.

3. Display information on the monitor screen in a convenient and human-readable form.

4. Maintenance of a real-time database with technological information.

5. Alarm and alarm management.

6. Realization of network interaction between SCADA PC.

\section{Literature}

[1] Abbas Hosny and Mohamed Ahmed. Efficient Web-Based SCADA system. M.: LAP Lambert Academic Publishing, 2012. - $124 \mathrm{c}$.

[2] Albahari Joseph, Albahari Ben. C\# 5.0 in a Nutshell: The Definitive Reference. - M.: O'Reilly Media, 2012. - 1068 c.

[3] Bondalapati Prasanth. ArcGIS and Databases. - M.: LAP Lambert Academic Publishing, 2011. - 80 c.
[4] Clarke Gordon. Practical Modern SCADA Protocols. 2010. - $544 \mathrm{c}$.

[5] Developer documentation for the ArcGIS Platform (https://developers.arcgis.com/documentation/)

[6] Harder Christian. ArcView GIS Means Business.

[7] Microsoft Developer Network (https://msdn.microsoft.com/en-us/)

[8] Peters Dave. Building a GIS: System Architecture Design Strategies for Managers. - 2008. - 292 c.

[9] Richter Jeffrey. CLR via C\#, Fourth Edition (October, 2012)

[10] Zahid Asghar Muhammad. Industrial Automation Using SCADA Based System. - M.: LAP Lambert Academic Publishing, 2012. $-72 \mathrm{c}$.

\section{About Authors}

Kabanov Peter Alexandrovich, student master information technology departments at the National Research Tomsk Polytechnic University. E-mail: Peter96pvl@gmail.com.

Sukhodoev Mikhail Sergeevich, candidate of Technical Sciences, associate professor of Automation and Robotics at the National Research Tomsk Polytechnic University. E-mail: smike@tpu.ru. 